OPEN ACCESS

Edited by:

Mingkai $L i$,

Fourth Military Medical University,

China

Reviewed by:

Filomena Nazzaro,

National Research Council (CNR), Italy

Lidija Senerovic,

University of Belgrade, Serbia

${ }^{*}$ Correspondence:

Weihua Chu

chuweihua@cpu.edu.cn

Specialty section:

This article was submitted to Molecular Bacterial Pathogenesis,

a section of the journa

Frontiers in Cellular and Infection

Microbiology

Received: 24 July 2020 Accepted: 05 October 2020 Published: 30 October 2020

Citation:

Tang J, Wang W and Chu W (2020) Antimicrobial and Anti-Quorum Sensing Activities of Phlorotannins From Seaweed (Hizikia fusiforme). Front. Cell. Infect. Microbiol. 10:586750. doi: 10.3389/fcimb.2020.586750

\section{Antimicrobial and Anti-Quorum Sensing Activities of Phlorotannins From Seaweed (Hizikia fusiforme)}

\author{
Jiali Tang, Wenqian Wang and Weihua $\mathrm{Chu}^{1 *}$ \\ School of Life Science and Technology, State Key Laboratory of Natural Medicines, China Pharmaceutical University, \\ Naniing, China
}

Multidrug-resistant bacteria (MDR) are becoming a global health problem, and scientists are continuously investigating new strategies to fight against MDR. Seaweeds are an important source of biological compounds and can serve as natural sources for bacterial infection control. This study evaluated the antimicrobial and anti-quorum sensing (QS) activities of phlorotannins from Hizikia fusiforme. The phlorotannins exhibited antimicrobial activity against selected bacterial pathogens and inhibited QS activity of the reporter strain Chromobacterium violaceum 12472 by inhibiting purple pigment production. Phlorotannins can decrease the bacterial motility, reduce the production of extracellular protease, hemolysin, and pyocyanin and inhibit biofilm formation of Pseudomonas aeruginosa. In vivo studies showed that phlorotannins can reduce $P$. aeruginosa inflicted mortality in Caenorhabditis elegans. This study shows that phlorotannins from H. fusiforme have certain antimicrobial and anti-quorum sensing activities and have the potential to control bacterial infection for pharmaceutical usage.

Keywords: phlorotannins, anti-quorum sensing, virulence factors, biofilm, Pseudomonas aeruginosa, Caenorhabditis elegans, anti-infection

\section{INTRODUCTION}

Antibiotics have been used for bacterial infection treatment for decades, with the majority used as feed additives for animal husbandry (Van Boeckel et al., 2017). However, antibiotics are becoming ineffective because of the emergence of antibiotic-resistant strains of bacteria (Mazer-Amirshahi et al., 2017; Ghosh et al., 2019; Mulani et al., 2019). The emergence and spread of multidrugresistant bacteria (MDR) have potentially profound consequences for public health in low- and middle-income countries (Van Boeckel et al., 2019). Thus, scientists have made efforts to find new agents and alternative antibiotics to work against bacterial pathogens (Czaplewski et al., 2016; Romero-Calle et al., 2019).

Quorum sensing (QS) is a cell-to-cell communication pathway in microorganisms, in which the expression of several genes, often associated with virulence factors and biofilm formation, is controlled via the production and detection of signal molecules in a population density-dependent manner (Rutherford and Bassler, 2012). Furthermore, signal disruption referred to as quorum quenching has been described in several biological systems and is being explored as a novel approach to fight bacterial infections. Disruption of QS is a competition strategy used by microorganisms and higher organisms (Defoirdt, 2018; Fleitas Martínez et al., 2019). 
Seaweeds are non-vascular, photosynthetic plants that inhabit near-coastal regions and have been one of the richest and most promising sources of primary and secondary bioactive metabolites with antimicrobial properties (Pérez et al., 2016; Zerrifi et al., 2018). Phlorotannins are polyphenolic compounds of seaweeds and have many biological activities including antioxidant, antidiabetic, anti-inflammatory, and antimicrobial (Javed et al., 2020). In this study, we investigated the effects of phlorotannins from brown seaweed (Hizikia fusiforme) on QS activity in Chromobacterium violaceum and virulence factors and biofilm formation in Pseudomonas aeruginosa. Furthermore, we determined the survival rate of Caenorhabditis elegans against $P$. aeruginosa infection.

\section{MATERIALS AND METHODS}

\section{Chemicals and Reagents}

Phlorotannins were purchased from the Shandong Jiejing Group Corporation (Shandong, China), which were isolated from Hizikia fusiforme. The phlorotannins were dissolved in water and stored at $4^{\circ} \mathrm{C}$ for further experiments. All other chemicals were of reagent grade from commercial sources.

\section{Biosensors and Culture Conditions}

Chromobacterium violaceum ATCC 12472, which can produce violacein (purple pigment) under the QS system, was used as a report strain to detect QS inhibition ability (Mclean et al., 2004). The reporter bacterium Agrobacterium tumefaciens A136 (pCF218)(pCF372) was used to detect the AHLs of Pseudomonas aeruginosa. The antimicrobial activity of phlorotannins was determined against Staphylococcus aureus ATCC 25923, Escherichia coli ATCC 25922, Pseudomonas aeruginosa PAO1, Aeromonas hydrophila YJ-1, and Vibrio parahaemolyticus X-1.

All bacteria were grown in Luria-Bertani broth (LBB, 0.5\% yeast extract, $1.0 \%$ tryptone, and $0.5 \% \mathrm{NaCl}$, at $\mathrm{pH} 7.4) . P$. aeruginosa, S. aureus, and E. coli were grown at $37^{\circ} \mathrm{C} ; \mathrm{C}$. violaceum, A. hydrophila, and $V$. parahaemolyticus were grown at $30^{\circ} \mathrm{C}$. Both the sets of microorganisms were incubated at 120 rpm for 18-24 h. The bacterial density was measured using a spectrophotometer at a wavelength of $600 \mathrm{~nm}\left(\mathrm{OD}_{600}\right)$. For long term storage, bacterial cultures were preserved at $-70^{\circ} \mathrm{C}$ in $\mathrm{LBB}$ containing 20\% (v/v) glycerol.

Caenorhabditis elegans N2 (Bristol) was obtained from the Caenorhabditis Genetics Center (CGC). The N2 strain was propagated under standard conditions, synchronized using hypochlorite bleaching, and cultured on nematode growth medium (NGM) at $16-25^{\circ} \mathrm{C}$ using an E. coli strain OP50 as a standard food source.

\section{AHLs Extraction and Analytical Thin-Layer Chromatography}

To evaluate the profiles of AHLs, bacterial culture supernatants were extracted and subjected to analytical thin-layer chromatography (TLC) (Shaw et al., 1997). A 10-ml sample of culture supernatant was extracted twice with equal volumes of ethyl acetate and then dried in a fume hood. The residues of extraction were then dissolved in $100 \mu \mathrm{l}$ of HPLC-grade ethyl acetate. Analytical TLC was performed on C18 reversed-phase TLC plates (Whatman, Clifton, NJ, USA). Chromatograms were developed with methanol: water (60:40, v:v) then air-dried in a fume hood. TLC plates were overlaid with a culture of the reporter bacterium A. tumefaciens A136 (pCF218)(pCF372) seeded in a thin layer of agar containing X-Gal. This traG:: lacZ/traR reporter detects 3-oxo-substituted AHL derivatives with acyl chain length from 4 to 12 carbons (e.g., OdDHL). After incubation of the plate at $30^{\circ} \mathrm{C}$ for $24 \mathrm{~h}$, AHLs were located as green spots on a white background. The development of blue spots indicated the induction of $\beta$-galactosidase expression in the reporter strain caused by the presence of AHLs. All the experiments were performed at least twice.

\section{Determination of Minimum Inhibitory Concentration}

The phlorotannins MIC for the selected bacteria was determined using a twofold dilution method in LBB with an inoculum of $10^{6}$ $\mathrm{CFU} / \mathrm{ml}$. After culturing at $37^{\circ} \mathrm{C}$ for $18 \mathrm{~h}$, the clarity of each tube was observed. The MIC was defined as the lowest concentration at which there is no visible bacteria growth in the tube (Wiegand et al., 2008). The sub-MIC (concentrations below MIC value) of phlorotannins was taken to evaluate the anti-QS activity on $P$. aeruginosa.

The synergistic effect of antibiotics (Levofloxacin and Amikacin) in combination with phlorotannins was tested by the chequerboard titration method as described by He et al. (2014). The final concentrations of the drugs were prepared in a range of $1 / 16-2 \times$ MIC. The fractional inhibitory concentration index (FICI) was equal to (MIC of antibiotics in combination/MIC of antibiotics alone) + (MIC of phlorotannins in combination/MIC of phlorotannins alone). The definitions of synergism, indifference and antagonism were interpreted as FICI $\leq 0.5,>0.5$ and $\leq 4.0$, and $>4.0$, respectively.

\section{Bioassay for QS Inhibition Activity}

C. violaceum 12472 was used as the biosensor to detect anti-QS activity. Ten milliliters of $50^{\circ} \mathrm{C}$ warm molten LB soft agar $(0.7 \%$ agar) was seeded with $20 \mu \mathrm{l}$ of $18 \mathrm{~h}$ cultured C. violaceum 12472 and mixed. The mixed culture was poured over the surface of a solidified LB plate to form the overlay. Subsequently, $50 \mathrm{~mm}$ wells were punched through the agar, and $100 \mu \mathrm{l}$ of phlorotannins was loaded into each well. The plates were then cultured at $30^{\circ} \mathrm{C}$ for $16-18 \mathrm{~h}$. The absence of the purple violacein pigmentation of the bacterial lawn surrounding the well would indicate QS inhibition activity (Chenia, 2013).

\section{Attenuation of $P$. aeruginosa PAO1 Virulence Factors, Motility, and Biofilm Formation Motility Assay}

LB medium with 0.3 and $0.5 \%$ (w/v) Difco Bacto-agar was used to characterize the swimming and swarming motility of 
$P$. aeruginosa. The overnight cultures of $P$. aeruginosa PAO1 were inoculated onto the center of the plates with different subMIC phlorotannins using a sterile toothpick. The plates were then incubated at $37^{\circ} \mathrm{C}$ for $16-18 \mathrm{~h}$, and the motility was assessed by measuring the migration of bacteria from the inoculation point (Yang et al., 2017). The motility was detected in triplicate.

\section{Proteolytic and Hemolytic Activities and Pyocyanin Production}

Pyocyanin is a blue-redox extracellular product, which is controlled by rhlR-rhlI, lasR-lasI, and PQS QS systems in P. aeruginosa (Liang et al., 2008; Wiegand et al., 2008). An overnight culture of $P$. aeruginosa PAO1 cultured with different sub-MIC phlorotannins was centrifuged at 10,000 $\times \mathrm{g}$ for $15 \mathrm{~min}$. The supernatant was filtered with a $0.45 \mu \mathrm{m}$ sterilized film. The supernatant was used for extracellular proteolytic and hemolytic activities and pyocyanin production assay. The proteolytic activity was detected by the method of Nicodème et al. (2005), using 1.0\% skim milk as a substrate. The hemolytic activity test was performed using $5 \%(\mathrm{v} / \mathrm{v})$ sheep blood in a nutrient agar plate (Castro-Escarpulli et al., 2003). Each measurement was conducted in triplicate. Pyocyanin production was measured using the methods of Michel et al. (2002). Two milliliters of cell-free supernatants were extracted with $1.5 \mathrm{ml}$ chloroform and vortexed immediately. After centrifugation $(10,000 \times \mathrm{g}, 5 \mathrm{~min})$ the top layer was transferred to a new tube where $1.5 \mathrm{ml} 0.2 \mathrm{M} \mathrm{HCl}$ was added, and the color of the liquid changed to pink. The absorbance was the detected at $\mathrm{OD}_{520}$.

\section{Biofilm Formation Assay}

Quantifying biofilm formation of $P$. aeruginosa was detected as previously described by Agarwala et al. (2014). Using $10 \mu \mathrm{l}$ of 18 h cultured $P$. aeruginosa PAO1 $\left(10^{6} \mathrm{CFU} / \mathrm{ml}\right)$, diluted with $2 \mathrm{ml}$ LB liquid medium containing a series of sub-MIC phlorotannins, incubation was conducted for $18 \mathrm{~h}$ in a static culture. The culture was discarded after incubation, and the tube was washed thrice with Phosphate Buffered Saline (PBS) and fixed in formaldehyde (10\%) for $10 \mathrm{~min}$. The solutions were removed, air dried at room temperature, and crystal violet ( $0.1 \%$ in ethanol) was added to stain the biofilm for $15 \mathrm{~min}$. Deionized water was used to wash the unbound dye three times, and the absorbed dye was eluted with ethanol. Then the $\mathrm{OD}_{590}$ was recorded, repeated independently three times.

The biofilm formation on coverslips was observed as described by Mishra et al. (2020). Biofilm of P. aeruginosa PAO1 was formed on the coverslips $(10 \times 10 \mathrm{~mm})$ in 12 -wells polystyrene microtiter plates (Liu et al., 2019). Different sub-MIC of phlorotannins was added. The inoculated broths were then cultivated for $24 \mathrm{~h}$. The coverslips were washed gently with PBS to remove the un-attached cells. For light microscopic analysis, the coverslips were stained with $0.4 \%$ crystal violet (CV) for 10 min. For fluorescence microscope analysis, the coverslips were stained with Live \& Dead Bacterial Staining Kit (Shanghai Yeasen Biotech Co. Ltd., Shanghai, China) at room temperature for $15 \mathrm{~min}$ in the dark and then washed with PBS to remove excess stain. The coverslips were allowed to dry and visualized under respective microscopes.

\section{C. elegans Killing Assay}

NGM was used to culture the C. elegans $\mathrm{N}_{2}$, and the protective effect of phlorotannins on $P$. aeruginosa PAO1 infection was evaluated. Treatment plates with different concentrations of phlorotannins were prepared by spreading $100 \mu \mathrm{l}$ of overnight cultured PAO1. The plate was incubated at $37^{\circ} \mathrm{C}$ for $16-18 \mathrm{~h}$ to grow a bacterial lawn, and $E$. coli OP50 plates were used as a negative control. About 20-30 synchronized L4 worms were transferred to each plate with the bacterial lawn, and the number of worms killed was recorded over 6 days using visual observation of the plates under a microscope (Kirienko et al., 2014).

\section{Statistical Analysis}

All tests were conducted in triplicate and data were presented as the mean values. Analysis of variance was conducted, and differences between the mean values were evaluated for significance using one-way ANOVA with Tukey test correction on the SPSS Statistics 20.0 and Origin Pro 8.0. Differences with a $p<0.05$ were considered statistically significant.

\section{RESULTS}

\section{Antibacterial and Anti-QS Activities Assays}

The phlorotannins' antibacterial activity was investigated using a double broth dilution method, and the minimal inhibitory concentration value was calculated. The MIC of phlorotannins against the selected bacteria was: $0.1943 \mathrm{~g} / \mathrm{ml}$ for C. violaceum and E. coli; whereas it was $0.0972 \mathrm{~g} / \mathrm{ml}$ for S. aureus, A. hydrophilia, $V$. parahaemolyticus, and $P$. aeruginosa.

Therefore, phlorotannins show QS inhibitory activity in C. violaceum 12472. Thus, we used $1 / 2$ MIC to detect the antiQS effect; after a 16-18 h culture, a muddy zone without purple pigment diameter was observed (Figure 1), where the halo zone formation indicates anti-QS phlorotannin activity.

Figure 2 showed the growth curve of $P$. aeruginosa PAO1 in the presence of different sub-MICs of phlorotannins; it does not show any significant difference in the growth pattern as compared to phlorotannins' free group. That indicates that sub-MIC phlorotannins have no effect on the cell division.

Acyl-HSL signal profiles present in $P$. aeruginosa were examined by thin layer chromatography and acyl-HSLresponsive bioassays. We found that phlorotannins did affect acyl-HSL production by $P$. aeruginosa. From Figure 3, we can see one of the quorum sensing molecules were lost.

The synergistic effect of antibiotics in combination with phlorotannins was determined. As shown in Table 1, the combination of phlorotannins can decreased the MIC of levofloxacin and amikacin. The FICIs were 1.25 and 1.5 for levofloxacin and amikacin, respectively. Thus these results indicated that this combination did not act synergistically against $P$. aeruginosa $\mathrm{PAO}$. 


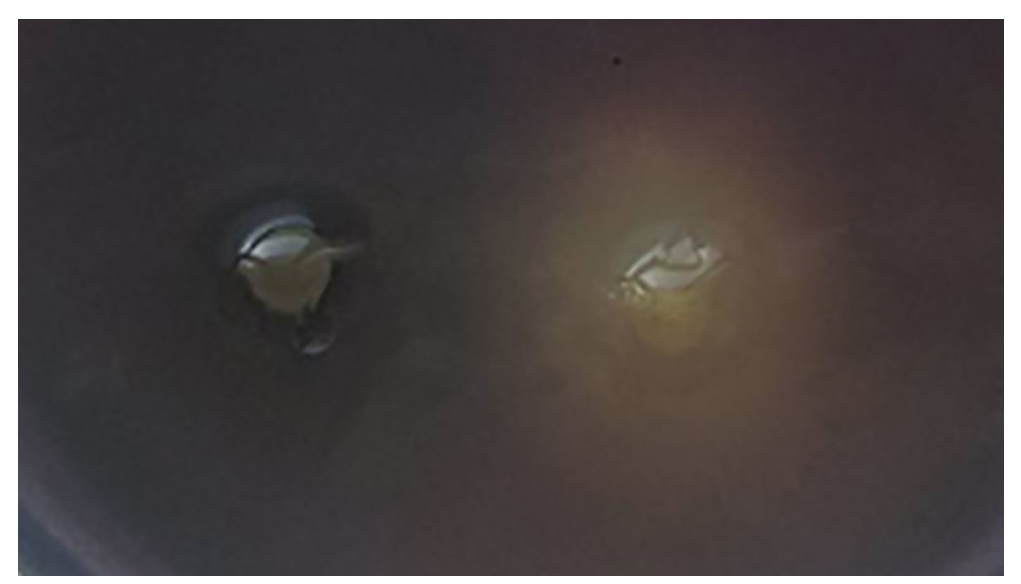

FIGURE 1 | Anti-QS activity of phlorotannins on a biosensor plate containing C. violaceum 12472.

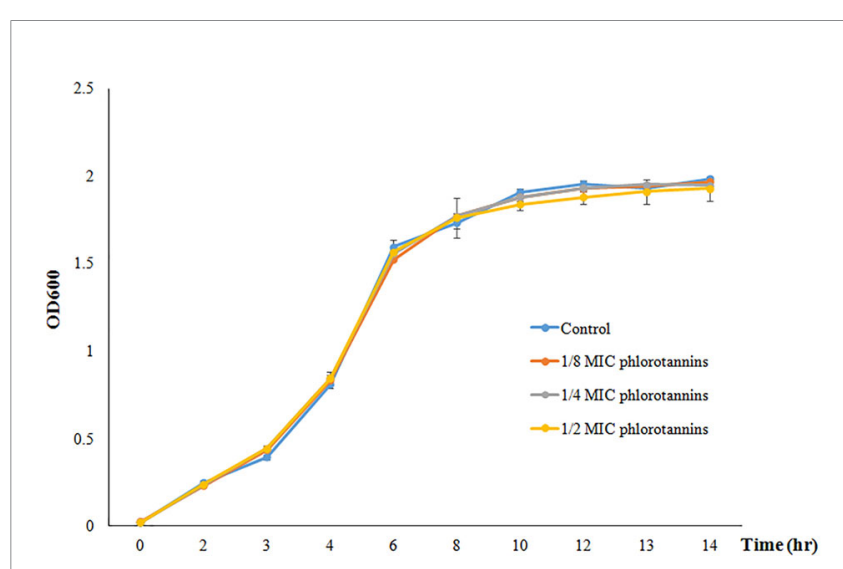

FIGURE 2 | Growth curve of $P$. aeruginosa PAO1 in the presence of different sub-MIC concentrations of phlorotannins (control, 1/8 MIC, 1/4 MIC, and 1/2 $\mathrm{MIC})$.

\section{Effect of Phlorotannins on Motility of $P$. aeruginosa PAO1}

Some phenotypes in P. aeruginosa PAO1, including bacterial motility, are controlled by QS systems; thus we detected the antimotility activities of phlorotannins on P. aeruginosa PAO1. Figure 4 shows phlorotannins can decrease the motility of $P$. aeruginosa because the diameter of the bacterial lawn decreased with the increasing phlorotannin concentration.

\section{Phlorotannins Suppress Proteolytic and Hemolytic Activities of $P$. aeruginosa PAO1}

The proteolytic and hemolytic activities were detected using skim milk and blood as substrates. The diameters of transparent zones were measured, which indicated the phlorotannins possess proteolytic and hemolytic activities (Table 2). Notably, with the increasing phlorotannin concentration, the transparent zone diameter increased. At the highest concentration tested $(1 / 2$ MIC, $0.04858 \mathrm{~g} / \mathrm{ml})$, the proteolytic activity of $P$. aeruginosa $\mathrm{PAO} 1$ reduced by 21.7 and $24.3 \%$, while hemolytic activity reduced by 11.0 and $21.8 \%$ when compared to untreated controls.

\section{Phlorotannins Decrease Pyocyanin Production of $\boldsymbol{P}$. aeruginosa PAO1}

Pyocyanin was produced when the cells of $P$. aeruginosa PAO1 reached high density, and production was limited when cultured with materials which can disrupt the QS systems but did not affect cell growth. Phlorotannins significantly reduced pyocyanin production; with the increasing concentration of phlorotannins, the pyocyanin production decreased (Figure 5).

\section{Phlorotannins Inhibit Biofilm Formation of P. aeruginosa PAO1}

The ability of phlorotannins to inhibit $P$. aeruginosa PAO1 biofilm formation activity was assessed using a crystal violet assay. As shown in Figure 6, a decrease in biofilm formation was observed when $P$. aeruginosa PAO1 was cultured with phlorotannins. Still, there were no statistically significant differences observed in reducing the biofilm formation at $0.0061-0.0121 \mathrm{~g} / \mathrm{ml}$. Similar results were also received by observing the biofilm with the help of light as well as fluorescent microscopy in Figure 7. It can be clearly observed that with the increase of phlorotannin concentration, the thinner biofilm appeared, which indicates intensive $P$. aeruginosa biofilm formation on coverslip surfaces. Viable bacteria cells were stained fluorescent green, whereas dead bacteria were stained fluorescent red. From the fluorescence micrograph images, we can find that with the increase of phlorotannin concentration, the green fluorescence and red fluorescence all decreased. Bacteria cell lysis at stationary phase, from Figure 2, we can find that at 10 h, $P$. aeruginosa cells were in the stationary growth phase; here we used 


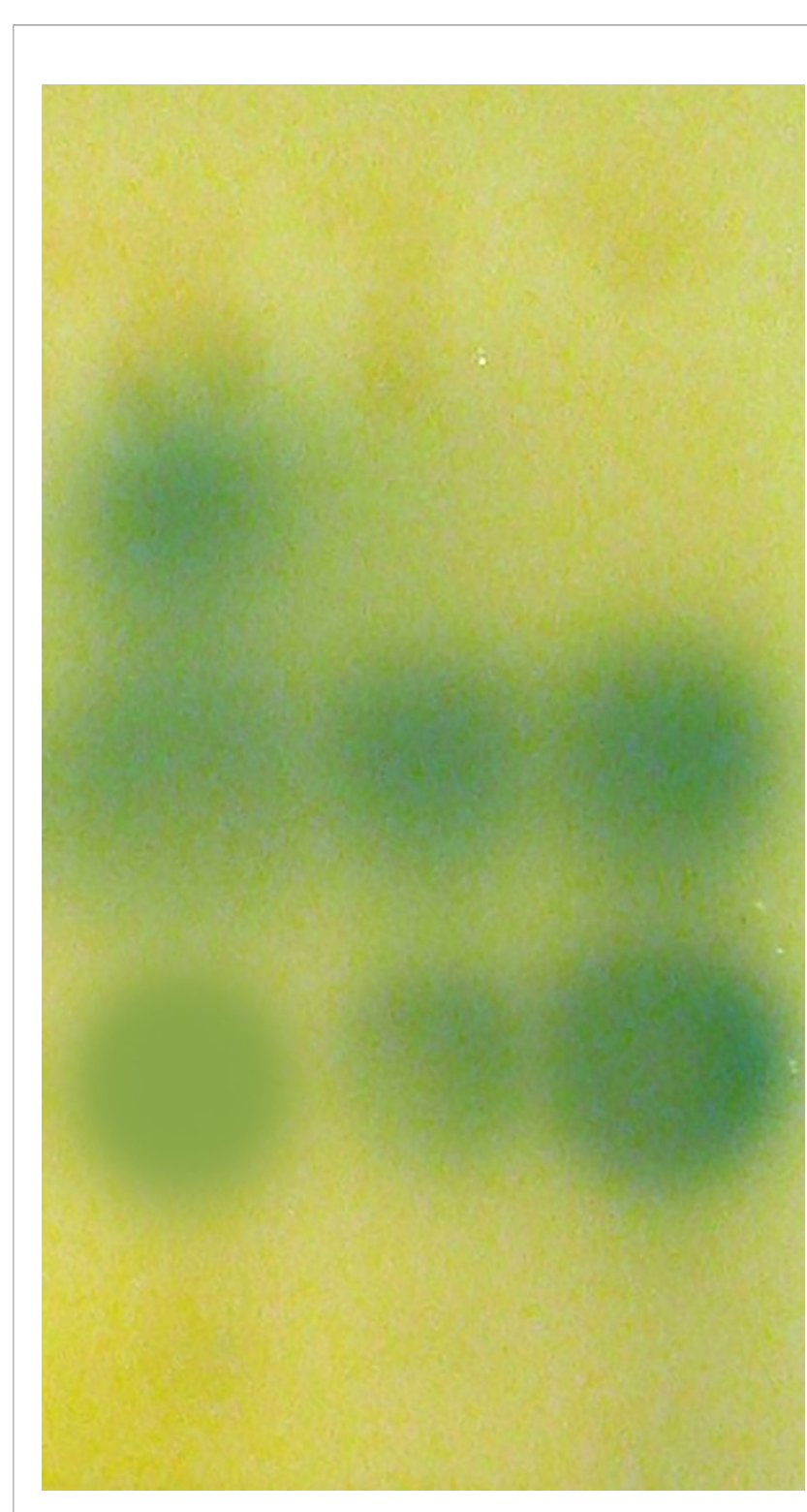

FIGURE 3 | Detection of AHLs by thin layer chromatography (TLC) with the A. tumefaciens A136 strain used as a biosensor. Lane 1, Extract of PAO1 culture, lane 2, Extract of PAO1 culture with 1/2 MIC phlorotannins, lane 3, Extract of PAO1 after culture with 1/4 MIC phlorotannins.

TABLE 1 | Effect of phlorotannins in combination with antibiotics against $P$. aeruginosa $\mathrm{PAO} 1$.

\begin{tabular}{|c|c|c|c|c|c|c|}
\hline \multirow[t]{2}{*}{ Antibiotics } & \multicolumn{2}{|c|}{$\begin{array}{l}\text { MIC of Antibiotics } \\
(\mathrm{mg} / \mathrm{L})\end{array}$} & \multicolumn{2}{|c|}{$\begin{array}{c}\text { MIC of } \\
\text { phlorotannins } \\
(\mathrm{g} / \mathrm{ml})\end{array}$} & \multirow[t]{2}{*}{$\mathrm{FICl}$} & \multirow[t]{2}{*}{ Activity } \\
\hline & Alone & $\begin{array}{l}\text { Combined } \\
\text { with } \\
\text { phlorotannins }\end{array}$ & Alone & $\begin{array}{l}\text { Combined } \\
\text { with } \\
\text { antibiotics }\end{array}$ & & \\
\hline Levofloxacin & 0.25 & 0.0625 & 0.0972 & 0.0972 & 1.25 & indifference \\
\hline Amikacin & 0.5 & 0.25 & 0.0972 & 0.0972 & 1.5 & indifference \\
\hline
\end{tabular}

$12 \mathrm{~h}$ culture to observe the biofilm, so the green fluorescence and red fluorescence all decreased at the same level. These results are in accordance with CV staining, which indicated the decreased biofilm biomass. Based on these results, it can be concluded that phlorotannins can inhibit biofilm formation.

\section{Phlorotannins Prevents C. elegans From Infection by $P$. aeruginosa PAO1}

Phlorotannins showed antibacterial and anti-QS activity in vitro; thus we detected their protective effect on C. elegans against $P$. aeruginosa $\mathrm{PAO}$ infection. Phlorotannins increased the survival of the infected C. elegans (Figure 8); furthermore, phlorotannins reduced nematode death in a concentrationdependent manner.

\section{DISCUSSION}

Virulence factors are required as a critical condition for bacterial infection. Therefore, anti-virulence strategies have been developed to prevent bacterial infection instead of killing it using antibiotics. Interruption of the QS is a potential strategy to attenuate pathogenicity because virulence factor expressions are controlled by QS in many bacteria. Phlorotannins are marine polyphenols derived from brown algae. Phlorotannins possess broad range of biological properties such as antimicrobial, antiviral, anticancer, antioxidant, anti-inflammatory, anti-allergic, and anti-hyperpigmentation properties (Audibert et al., 2010; Lemesheva and Tarakhovskaya, 2018; Javed et al., 2020). In this study, phlorotannins were evaluated for their antibacterial and anti-QS activities. We found phlorotannins have antibacterial activity against selected gram-positive and negative bacteria in vitro, but phlorotannins have no synergistic effect with selected antibiotics. Pérez et al. (2016) reviewed the antimicrobial action of compounds from marine seaweed and their application in biofouling, aquaculture, and human health. Furthermore, Moubayed et al. (2017) found Sargassum latifolium B, Sargassum platycarpum A (collected from the red sea), and Cladophora socialis (collected from the Arabian Gulf) demonstrated antibacterial activity. The methanolic extracts of Enteromorpha antenna, Enteromorpha linza, and Gracilaria corticata possessed high total phenolic content and showed antimicrobial activity against food-borne pathogens except $P$. aeruginosa (Narasimhan et al., 2013).

Plant phenolic compounds have been reported have anti-QS activities. Violacein production by C. violaceum 12472 is regulated by the QS system. Therefore, the reduced production of violacein in C. violaceum is evidence of QS disruption (Javed et al., 2020). In this study, we found phlorotannins can reduce the purple pigment production in C. violaceum 12472. Our experimental data also showed that phlorotannins could decrease the virulence factor's production and biofilm formation and also inhibit quorum sensing molecules production in $P$. aeruginosa. Moreover, phlorotannins also significantly improved the survival of 

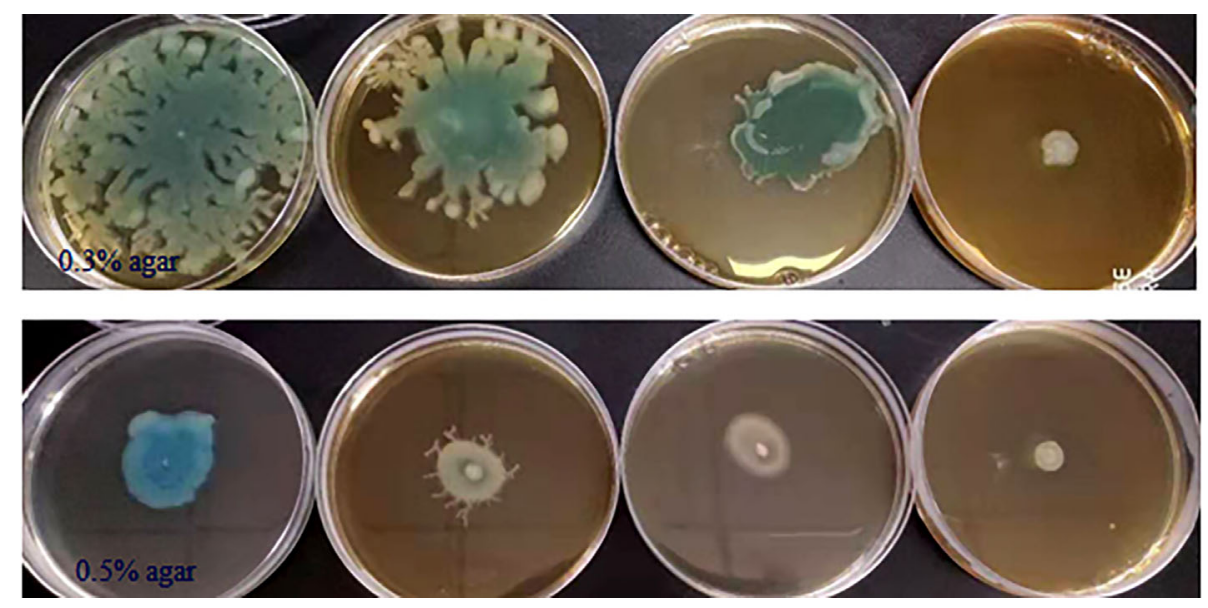

FIGURE 4 | Effect of phlorotannins on the motility of $P$. aeruginosa PAO1. P. aeruginosa PAO1 swimming motility and swarming motility on different concentrations of phlorotannins. From left to right: control, 1/8 MIC, 1/4 MIC, and 1/2 MIC.

TABLE 2 | Diameters of transparent zone for proteolytic and hemolytic activities.

\begin{tabular}{lccccc}
\hline \multicolumn{5}{c}{ Concentration (g/ml) } \\
\hline Phlorotannins & 0 & 0.00607 & 0.01214 & 0.02429 & 0.04858 \\
Protease & $1.52 \pm$ & $1.26 \pm$ & $1.21 \pm$ & $1.17 \pm$ & $1.15 \pm$ \\
& 0.0764 & 0.0529 & 0.0 .513 & 0.0289 & 0.0500 \\
Hemolysin & $1.19 \pm$ & $1.15 \pm$ & $1.10 \pm$ & $1.03 \pm$ & $0.93 \pm$ \\
& 0.0478 & 0.0600 & 0.0300 & 0.0353 & 0.0353 \\
\hline
\end{tabular}

Data are expressed as mean \pm SE of three separate independent experiments.

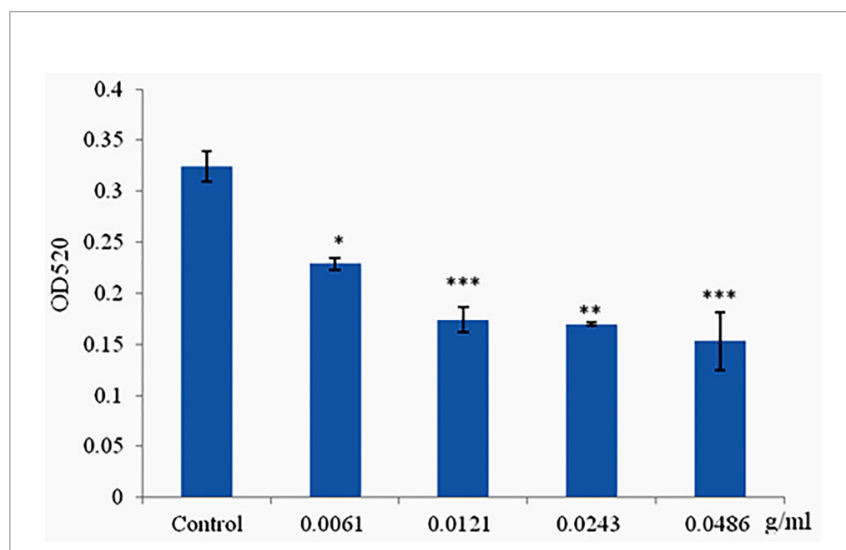

FIGURE 5 | Phlorotannins on pyocyanin production. Asterisks indicate a statistical difference between experimental groups and control groups. Results represent three independent experiments performed in triplicates. $\left({ }^{\star} p<0.05 ;{ }^{* *} p<0.01 ;{ }^{* \star *} p<0.001\right)$.

C. elegans against $P$. aeruginosa infection. Our results agree with previous reports. Tea polyphenol inhibited biofilm formation and swimming motility of Shewanella baltica and also decreased extracellular protelytic activity, exopolysaccharide production in S. baltica (Zhu et al., 2015). Biancalani et al.'s study showed that

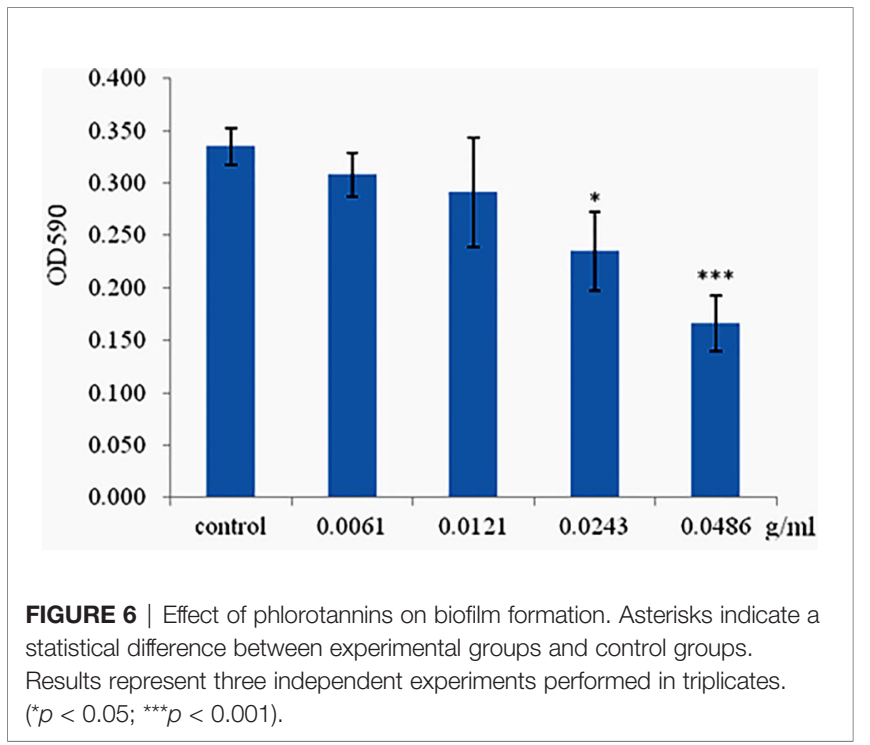

polyphenolic extracts from vegetable residues had a high inhibitory activity on QS of Pseudomonas savastanoi (Biancalani et al., 2016). Red seaweed (Chondrus crispus and Sarcodiotheca gaudichaudii) extracts can suppress the expression of QS gene sdiA, reduce biofilm formation and motility, and down regulate the expression of genes encoding the virulence factors of Salmonella enterica. Red seaweed extract significantly increased the survival of the infected C. elegans (Kulshreshtha et al., 2016). Another study by Carvalho et al. (2017) showed that Canistrocarpus cervicornis extracts could inhibit QS of C. violaceum CV017 and inhibit bacterial attachment of $P$. aeruginosa PAO1. Jha et al. (2013) found that the extract from marine macro alga (Asparagopsis taxiformis) has QS inhibition properties, and the expected active compound was 2-dodecanoyloxyethanesulfonate $\left(\mathrm{C}_{14} \mathrm{H}_{27} \mathrm{O}_{5} \mathrm{~S}\right)$. 


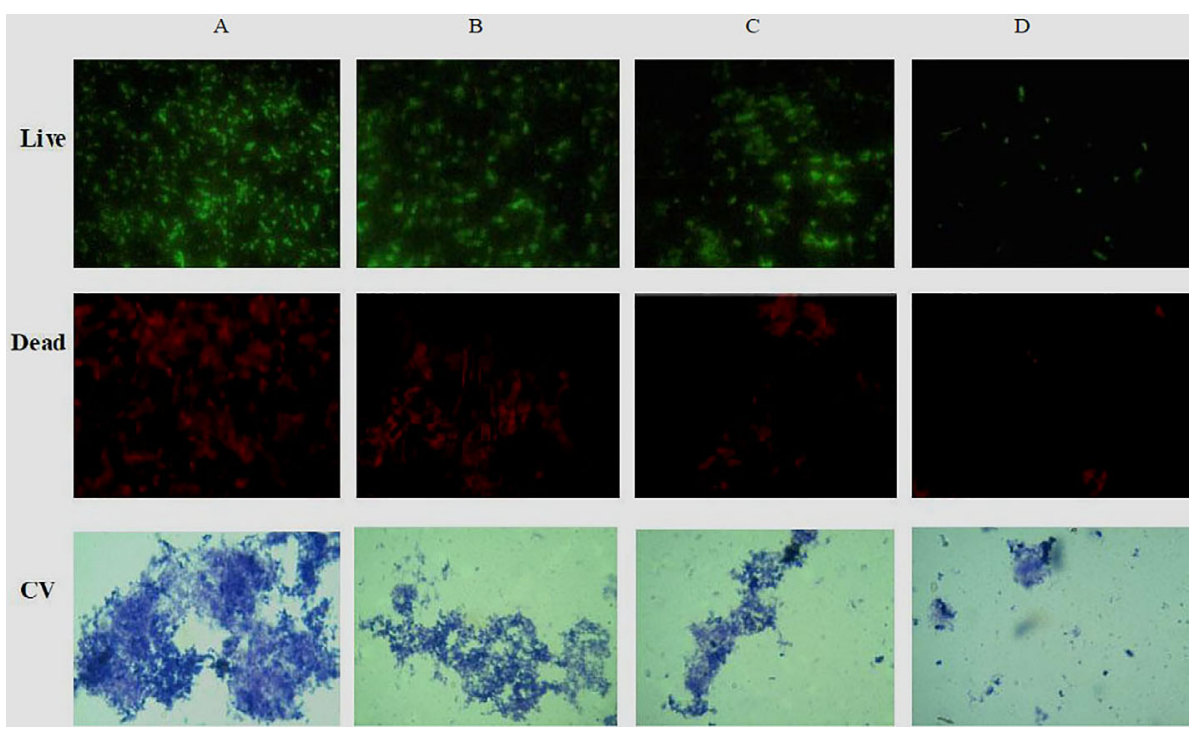

FIGURE 7 | Fluorescence and light microscopic images of biofilms when treated with various concentrations of phlorotannins. (A) control, (B-D) demonstrated 1/8, $1 / 4$ and $1 / 2$ MIC phlorotannins treated, respectively.

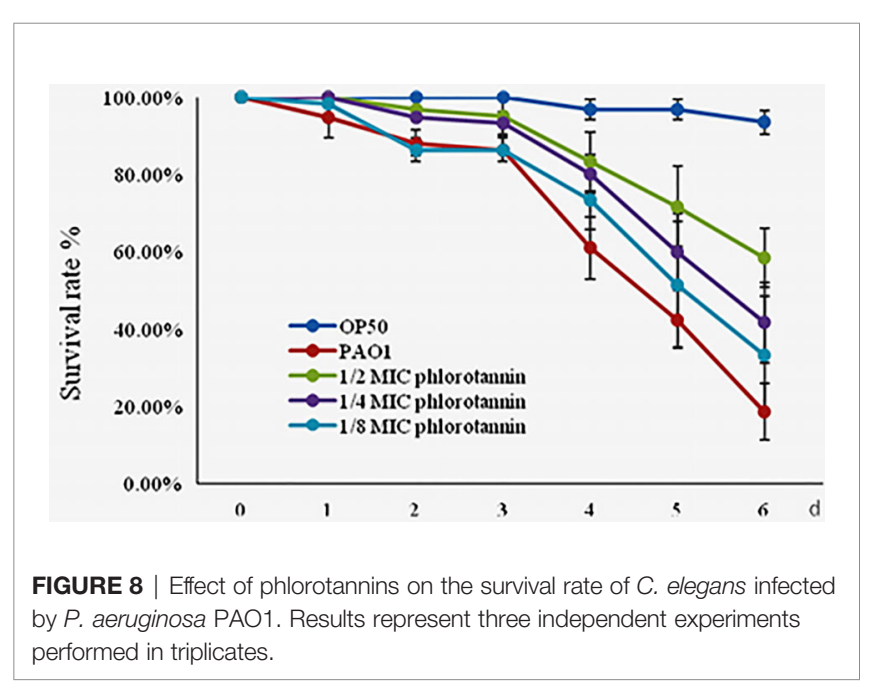

Batista et al. (2014) showed that 20 polar extracts of macroalgae from Arraial do Cabo, Brazil, interrupted the QS of the reporter C. violaceum. Halogenated furanones from the marine algae Delisea pulchra inhibit AHLs-mediated quorum sensing (Gram et al., 1996). Three AHL inhibitors have been isolated from the Korean red alga Ahnfeltiopsis flabelliformis (Kim et al., 2007).

Based on our results, it is proven that phlorotannins possess anti-QS activities and can attenuate virulence determinants of $P$. aeruginosa. However, future investigations should use the phlorotannins in animal feed to evaluate its clinical effect.

In conclusion, phlorotannins can inhibit the QS activity, reduce virulence factor's production, and disrupt biofilm formation, which is crucial to the pathogenicity in $P$. aeruginosa. Phlorotannins present its use as an important natural source for controlling bacterial infections in the future. This method provides a viable alternative strategy to antibiotics against pathogens, without selective pressure on the drug resistant mutant strains and can be used in pharmaceutical industries.

\section{DATA AVAILABILITY STATEMENT}

The original contributions presented in the study are included in the article/supplementary material. Further inquiries can be directed to the corresponding author.

\section{AUTHOR CONTRIBUTIONS}

WC designed the research. JT and WW performed sampling and performed experimental work. WW performed data analysis. WC wrote the manuscript and input from all authors. All authors contributed to the article and approved the submitted version.

\section{FUNDING}

This research was funded by the Priority Academic Program Development of Jiangsu Higher Education Institutions (PAPD). 


\section{REFERENCES}

Agarwala, M., Choudhury, B., and Yadav, R. N. S. (2014). Comparative study of antibiofilm activity of copper oxide and iron oxide nanoparticles against multidrug resistant biofilm forming uropathogens. Indian J. Microbiol. 54, 365-368. doi: 10.1007/s12088-014-0462-z

Audibert, L., Fauchon, M., Blanc, N., Hauchard, D., and Ar Gall, E. (2010). Phenolic compounds in the brown seaweed Ascophyllum nodosum: Distribution and radical-scavenging activities. Phytochem. Anal. 21, 399-405. doi: $10.1002 /$ pca. 1210

Batista, D., Carvalho, A. P., Costa, R., Coutinho, R., and Dobretsov, S. (2014). Extracts of macroalgae from the Brazilian coast inhibit bacterial quorum sensing. Bot. Mar. 57, 441-447. doi: 10.1515/bot-2014-0031

Biancalani, C., Cerboneschi, M., Tadini-Buoninsegni, F., Campo, M., Scardigli, A., Romani, A., et al. (2016). Global analysis of type three secretion system and quorum sensing inhibition of Pseudomonas savastano $i$ by polyphenols extracts from vegetable residues. PLoS One 11, e0163357. doi: 10.1371/journal. pone. 0163357

Carvalho, A. P., Batista, D., Dobretsov, S., and Coutinho, R. (2017). Extracts of seaweeds as potential inhibitors of quorum sensing and bacterial growth. J. Appl. Phycol. 29, 789-797. doi: 10.1007/s10811-016-1014-1

Castro-Escarpulli, G., Figueras, M. J., Aguilera-Arreola, G., Soler, L., and Chacón, M. R. (2003). Characterisation of Aeromonas spp. isolated from frozen fish intended for human consumption in Mexico. Int. J. Food Microbiol. 84, 41-49. doi: 10.1016/S0168-1605(02)00393-8

Chenia, H. Y. (2013). Anti-quorum sensing potential of crude Kigelia africana fruit extracts. Sensors 13, 2802-2817. doi: 10.3390/s130302802

Czaplewski, L., Bax, R., Clokie, M., Dawson, M., Fairhead, H., Fischetti, V. A., et al. (2016). Alternatives to antibiotics-a pipeline portfolio review. Lancet Infect. Dis. 16, 239-251. doi: 10.1016/S1473-3099(15)00466-1

Defoirdt, T. (2018). Quorum-sensing systems as targets for antivirulence therapy. Trends Microbiol. 26, 313-328. doi: 10.1016/j.tim.2017.10.005

Fleitas Martínez, O., Rigueiras, P. O., Pires, Á., da, S., Porto, W. F., Silva, O. N., et al. (2019). Interference with quorum-sensing signal biosynthesis as a promising therapeutic strategy against multidrug-resistant pathogens. Front. Cell Infect. Microbiol. 8, 444. doi: 10.3389/fcimb.2018.00444

Ghosh, C., Sarkar, P., Issa, R., and Haldar, J. (2019). Alternatives to conventional antibiotics in the era of antimicrobial resistance. Trends Microbiol. 27, 323338. doi: 10.1016/j.tim.2018.12.010

Gram, L., de Nys, R., Maximilien, R., Givskov, M., Steinberg, P., and Kjelleberg, S. (1996). Inhibitory effects of secondary metabolites from the red algae Delisea pulchra on swarming motility of Proteus mirabilis. Appl. Environ. Microbiol. 62, 4284-4287. doi: 10.1016/S0027-5107(96)00126-1

He, X., Hwang, H., Aker, W. G., Wang, P., Lin, Y., Jiang, X., et al. (2014). Synergistic combination of marine oligosaccharides and azithromycin against Pseudomonas aeruginosa. Microbiol. Res. 169, 759-767. doi: 10.1016/ j.micres.2014.01.001

Javed, A., Hussain, M. B., Tahir, A., Waheed, M., Anwar, A., Shariati, M. A., et al. (2020). Pharmacological applications of phlorotannins: a comprehensive review. Curr. Drug Discov. Technol. doi: 10.2174/1570163817666200206110243

Jha, B., Kavita, K., Westphal, J., Hartmann, A., and Schmitt-Kopplin, P. (2013). Quorum sensing inhibition by Asparagopsis taxiformis, a marine macro alga: separation of the compound that interrupts bacterial communication. Mar. Drugs 11, 253-265. doi: 10.3390/md11010253

Kim, J. S., Kim, Y. H., Seo, Y. W., and Park, S. (2007). Quorum sensing inhibitors from the red alga, Ahnfeltiopsis flabelliformis. Biotechnol. Bioprocess Eng. 12, 308-311. doi: 10.1007/BF02931109

Kirienko, N. V., Cezairliyan, B. O., Ausubel, F. M., and Powell, J. R. (2014). Pseudomonas aeruginosa PA14 pathogenesis in Caenorhabditis elegans. Methods Mol. Biol. 1149, 653-669. doi: 10.1007/978-1-4939-0473-0_50

Kulshreshtha, G., Borza, T., Rathgeber, B., Stratton, G. S., Thomas, N. A., Critchley, A., et al. (2016). Red Seaweeds Sarcodiotheca gaudichaudii and Chondrus crispus down regulate virulence factors of Salmonella Enteritidis and induce immune responses in Caenorhabditis elegans. Front. Microbiol. 7, 421. doi: $10.3389 /$ fmicb.2016.00421

Lemesheva, V., and Tarakhovskaya, E. (2018). Physiological functions of phlorotannins. Bio. Commun. 63, 70-76. doi: 10.21638/spbu03.2018.108
Liang, H., Li, L., Dong, Z., Surette, M. G., and Duan, K. (2008). The YebC family protein PA0964 negatively regulates the Pseudomonas aeruginosa quinolone signal system and pyocyanin production. J. Bacteriol. 190, 6217-6227. doi: 10.1128/JB.00428-08

Liu, Y., Jiang, Y., Zhu, J., Huang, J., and Zhang, H. (2019). Inhibition of bacterial adhesion and biofilm formation of sulfonated chitosan against Pseudomonas aeruginosa. Carbohyd. Polym. 206, 412-419. doi: 10.1016/j.carbpol.2018.11.015

Mazer-Amirshahi, M., Pourmand, A., and May, L. (2017). Newly approved antibiotics and antibiotics reserved for resistant infections: implications for emergency medicine. Am. J. Emerg. Med. 35, 154-158. doi: 10.1016/j.ajem.2016.10.034

Mclean, R. J. C., Pierson, L. S., and Fuqua, C. (2004). A simple screening protocol for the identification of quorum signal antagonists. J. Microbiol. Methods 58, 351-360. doi: 10.1016/j.mimet.2004.04.016

Michel, L., Harms, H., Heurlier, K., Michaux, P., Zala, M., Ginet, N., et al. (2002). Genetically programmed autoinducer destruction reduces virulence gene expression and swarming motility in Pseudomonas aeruginosa PAO1. Microbiology 148, 923-932. doi: 10.1099/00221287-148-4-923

Mishra, R., Kushveer, J. S., Khan, M. I. K., Pagal, S., Meena, C. K., Murali, A., et al. (2020). 2,4-Di-Tert-Butylphenol isolated from an endophytic fungus, Daldinia eschscholtzii, reduces virulence and quorum sensing in Pseudomonas aeruginosa. Front. Microbiol. 11, 1668. doi: 10.3389/fmicb.2020.01668

Moubayed, N. M. S., Al Houri, H. J., Al Khulaifi, M. M., and Al Farraj, D. A. (2017). Antimicrobial; antioxidant properties and chemical composition of seaweeds collected from Saudi Arabia (Red Sea and Arabian Gulf). Saudi J. Biol. Sci. 24, 162-169. doi: 10.1016/j.sjbs.2016.05.018

Mulani, M. S., Kamble, E. E., Kumkar, S. N., Tawre, M. S., and Pardesi, K. R. (2019). Emerging strategies to combat eskape pathogens in the era of antimicrobial resistance: a review. Front. Microbiol. 10, 539. doi: 10.3389/fmicb.2019.00539

Narasimhan, M. K., Pavithra, S. K., Krishnan, V., and Chandrasekaran, M. (2013). In vitro analysis of antioxidant, antimicrobial and antiproliferative activity of Enteromorpha antenna, Enteromorpha linza and Gracilaria corticata extracts. Jundishapur J. Nat. Pharm. Prod. 8, 151-159. doi: 10.0000/PMID24624206

Nicodème, M., Grill, J. P., Humbert, G., and Gaillard, J. L. (2005). Extracellular protease activity of different Pseudomonas strains: dependence of proteolytic activity on culture conditions. J. Appl. Microbiol. 99, 641-648. doi: 10.1111/ j.1365-2672.2005.02634.x

Pérez, M. J., Falqué, E., and Domínguez, H. (2016). Antimicrobial action of compounds from marine seaweed. seaweed bioactive compounds against pathogens and microalgae: potential uses on pharmacology and harmful algae bloom control. Mar. Drugs 14, 52. doi: 10.3390/md14030052

Romero-Calle, D., Guimarães Benevides, R., Góes-Neto, A., and Billington, C. (2019). Bacteriophages as alternatives to antibiotics in clinical care. Antibiot. (Basel). 8, 138. doi: 10.3390/antibiotics8030138

Rutherford, S. T., and Bassler, B. L. (2012). Bacterial quorum sensing: its role in virulence and possibilities for its control. Cold Spring Harbor Perspect. Med. 2 705-709. doi: 10.1101/cshperspect.a012427

Shaw, P. D., Ping, G., Daly, S. L., Ch,a, C., Cronan, J. E.Jr, Rinehart, K. L., et al. (1997). Detecting and characterizing $\mathrm{n}$-acyl-homoserine lactone signal molecules by thinlayer chromatography. PNAS 94, 6036-6041. doi: 10.1073/pnas.94.12.6036

Van Boeckel, T. P., Glennon, E. E., Chen, D., Gilbert, M., Robinson, T. P., Grenfell, B. T., et al. (2017). Reducing antimicrobial use in food animals. Science 357, 1350-1352. doi: 10.1126/science.aao1495

Van Boeckel, T. P., Pires, J., Silvester, R., Zhao, C., Song, J., Criscuolo, N. G., et al. (2019). Global trends in antimicrobial resistance in animals in low- and middleincome countries. Science 365, 1350-1352. doi: 10.1126/science.aaw1944

Wiegand, I., Hilpert, K., and Hancock, R. E. W. (2008). Agar and broth dilution methods to determine the minimal inhibitory concentration (mic) of antimicrobial substances. Nat. Protoc. 3, 163-175. doi: 10.1038/nprot.2007.521

Yang, R., Guan, Y., Zhou, J., Sun, B., and Wang, Z. (2017). Phytochemicals from Camellia nitidissima Chi flowers reduce the pyocyanin production and motility of Pseudomonas aeruginosa PAO1. Front. Microbiol. 8, 2640. doi: 10.3389/ fmicb.2017.02640

Zerrifi, S. E. A., Khalloufi, F., Oudra, B., and Vasconcelos, V. (2018). Seaweed bioactive compounds against pathogens and microalgae: potential uses on pharmacology and harmful algae bloom control. Mar. Drugs 16, 55. doi: $10.3390 / \mathrm{md} 16020055$

Zhu, J., Huang, X., Zhang, F., Feng, L., and Li, J. (2015). Inhibition of quorum sensing, biofilm, and spoilage potential in Shewanella baltica by 
green tea polyphenols. J. Microbiol. 53, 829-836. doi: 10.1007/s12275015-5123-3

Conflict of Interest: The authors declare that the research was conducted in the absence of any commercial or financial relationships that could be construed as a potential conflict of interest.
Copyright $\odot 2020$ Tang, Wang and Chu. This is an open-access article distributed under the terms of the Creative Commons Attribution License (CC BY). The use, distribution or reproduction in other forums is permitted, provided the original author(s) and the copyright owner(s) are credited and that the original publication in this journal is cited, in accordance with accepted academic practice. No use, distribution or reproduction is permitted which does not comply with these terms. 